Article

\title{
Protective Effect of Aplysin Supplementation on Intestinal Permeability and Microbiota in Rats Treated with Ethanol and Iron
}

\author{
Yan $\mathrm{Ma}^{1}$, Ruiying $\mathrm{Li}^{1}$, Ying Liu ${ }^{2}$, Man Liu ${ }^{1}$ and Hui Liang ${ }^{1, *}$ \\ 1 Department of Human Nutrition, College of Public Health, Qingdao University, 38 Dengzhou Road, \\ Qingdao 266021, China; mayanaa@163.com (Y.M.); liruiying1104@163.com (R.L.); \\ lium_summer@126.com (M.L.) \\ 2 Basic Medical College, Qingdao University of Medicine, 308 Ningxia Road, Qingdao 266071, China; \\ shenghua005@163.com (Y.L.) \\ * Correspondence: qdlianghui@126.com; Tel.: +86-532-8381-2434
}

Received: 24 April 2018; Accepted: 22 May 2018; Published: 27 May 2018

\begin{abstract}
Aplysin, a kind of phytochemicals or phytonutrients, is purified from red alga Laurencia tristicha. The present study aims to investigate the influence of aplysin on changes of intestinal permeability and microbiota induced by excessive ethanol and iron. Thirty male rats were randomly divided into three groups (10/group): control group (normal saline); ethanol + iron group as EI treated with ethanol $(8-12 \mathrm{~mL} / \mathrm{kg} /$ day $)$ and iron $(1000 \mathrm{mg} / \mathrm{kg})$ in diet; EI supplemented with aplysin $(150 \mathrm{mg} / \mathrm{kg} /$ day) group as AEI; the trial lasts for 12 weeks. The result showed that levels of plasma endotoxin, fatty acid-binding protein 2, D-lactic acid, diamine oxidase were increased in rats in the EI group; and significantly decreased by $14 \%, 17 \%, 26 \%, 16 \%$, respectively $(p<0.05)$ in the AEI group after the 12-week aplysin treatment. Moreover, in the AEI group the amount of Escherichia coli and Bacteroides fragilis were higher, while the amount of Lactobacillus, Bifidobacterium and Clostridium were lower than those in the EI group. The expressions of iron transporters divalent-metal transporter 1(DMT1) and ferroportin 1(FPN1) were significantly upregulated in the EI group compared to those in the control group. In conclusion, aplysin could effectively improve intestinal permeability and intestinal flora disorder induced with excessive ethanol and iron.
\end{abstract}

Keywords: ethanol; iron; aplysin; intestinal permeability; microbiota

\section{Introduction}

In the human body, iron is an essential element, but excessive iron intake can result in excessive production of oxygen free radicals [1], causing damage to different tissues and organs [2]. Chronic excessive alcohol intake causes liver injury, and over half of the patients with advanced alcoholic cirrhosis exhibited with high iron content in the liver [3], and a third of patients with chronic alcohol consumption also presented increased iron stores in the liver [4].

It has been found that different changes in intestinal microbiota and dysbiosis are caused by alcohol consumption in humans and animals $[5,6]$. Alcohol and its metabolites in the small intestine encounter a physical barrier, which plays a crucial role in preventing invasive pathogens into the body and protecting barrier integrity $[7,8]$. Alcohol intake may destroy intestinal barrier defense and lead to intestinal physical disruption [8,9]. It is well known that alcohol intake causes damage to intestinal barrier function, which can increase the mucosa permeability to many macromolecules $[10,11]$. Moreover, the close association of clinical expression of hemochromatosis and excessive alcohol consumption explained that the co-factor effect of iron and alcohol can lead to oxidative stress and 
hepatic injury [12]. However, it is unclear that co-treatment with ethanol and iron affects the intestinal permeability and gut microbiota.

Aplysin, a brominated sesquiterpene, is extracted from one kind of red alga named Laurencia tristicha (Figure 1). It has been previously described that its molecular formula is $\mathrm{C} 15 \mathrm{H} 19 \mathrm{OBr}$ and it has a molecular weight of 295 [13]. Aplysin potent biological activities include antitumor, anti-inflammatory, immunostimulation and antioxidant activities [14]. Previous study in our lab has demonstrated that a high dose aplysin treatment $\left(150 \mathrm{mg} \mathrm{kg}^{-1}\right.$ day $\left.^{-1}\right)$ can significantly protect alcoholic liver injury [13] by repairing intestinal barrier function, normalizing fecal microbiota, and reducing inflammatory response and plasma endotoxin level in rats [15]. However, the potential effect of aplysin on the intestinal microflora and barrier function in rats exposed to iron and ethanol has not been explored.

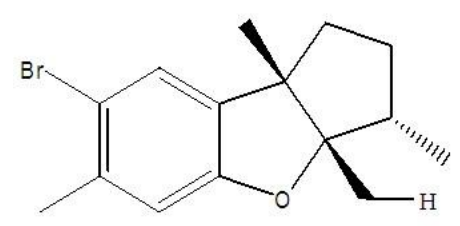

Figure 1. Chemical structure of aplysin.

Therefore, the present study aims to evaluate the effect of aplysin on the permeability of the intestine, the intestinal mucosal barrier, and intestinal microflora in rats exposed to iron and ethanol.

\section{Materials and Methods}

\subsection{Animal and Ethics Statement}

Animal Experiment Center (Qingdao, China) provided adult male Wistar rats weighting 180-220 g. The rats were kept on a $12 \mathrm{~h}$ light and $12 \mathrm{~h}$ dark at a relative humidity $(50-60 \%)$ and a controlled temperature $\left(22-25^{\circ} \mathrm{C}\right)$. Standard rodent diet was fed to animals during the whole study. The animals were cared for according to international guidelines for the use and care of Laboratory Animals (Institute of Laboratory Animal Resources, Commission on Life Science, National Research Council, 1996). The animal use and care committee of Medical College of Qingdao University approved the animal study.

\subsection{Experimental Designs and Animal Treatment}

After a 1-week period of acclimatization, 30 rats were randomly divided into 3 groups (10/group): C, control group, fed with normal saline + normal diet; EI, ethanol and iron model group, fed with normal diet contained dietary high iron $1000 \mathrm{mg} \mathrm{kg}^{-1}$ and gavaged with ethanol $56 \% \mathrm{v} / \mathrm{v}$ $\left(8 \mathrm{~mL} \mathrm{~kg}^{-1}\right.$ day $^{-1} 2$ week $+12 \mathrm{~mL} \mathrm{~kg}^{-1}$ day $^{-1} 10$ week); AEI, Aplysin treatment group, normal diet contained dietary high iron $1000 \mathrm{mg} \mathrm{kg}^{-1}$ and gavaged with ethanol and aplysin (the dose of ethanol was as same as that in model group, aplysin $150 \mathrm{mg} \mathrm{kg}^{-1} \mathrm{day}^{-1}$ ). The animals were supplemented with soya bean salad oil ( $1 \mathrm{~mL})$ through gavage for 12 weeks in control group and model group. Rats were administrated by gavage with aplysin $150 \mathrm{mg} \mathrm{kg}^{-1}$ day $^{-1}$ (soluble in soya bean salad oil) in the aplysin-treated group, following alcohol or saline lavage with $2 \mathrm{~h}$ interval for 12 weeks. Food consumption and body weight were monitored once a week and daily, respectively.

Aplysin is liposoluble and it can be dissolved in ethanol or other organic solvents. The Institute of Oceanology, Chinese Academy of Sciences identified the purity of aplysin-97.6\%. Animal Experiment Center (Qingdao, China) provided the normal diet, which contained carbohydrates, protein, lipids, vitamins and other substances (Table 1$)$. The type of alcohol (56\% $(v / v)$ ethanol) used in this study was Red Star Erguotou (Beijing Red Star Co., Ltd., Beijing, China). 
After 12 weeks, the experiments used metabolic cages to collect the faeces of each rat after $12 \mathrm{~h}$ from the last intervention. Then the animals were sacrificed, samples of blood (or plasma), liver and small intestine tissues were taken and stored at $-80^{\circ} \mathrm{C}$. Fresh tissues of the liver and small intestine were quickly excised, and the parts were fixed in $10 \%$ formaldehyde and embedded in paraffin.

Table 1. Composition of the diets.

\begin{tabular}{cc}
\hline The Composition of the Diets & $\mathbf{( g / k g )}$ \\
\hline Sucrose & 500.0 \\
Casein & 200.0 \\
Corn starch & 150.0 \\
Cellulose & 50.0 \\
Corn oil & 50.0 \\
Mineral mix, AIN-76 (170,915) & 35.0 \\
Vitamin mix, AIN-76A (40,077) & 10.0 \\
DL-Methionine & 3.0 \\
Choline bitartrate & 2.0 \\
Ethoxyquin, antioxidant & 0.01 \\
\hline
\end{tabular}

\subsection{Histopathological Analysis}

The experiments processed formalin-fixed liver and small intestine tissues with hematoxylino-eosin (HE) staining, and examined morphological changes under light microscope (Olympus BX60, Japan). The ultrastructure of the intestinal tissue was observed by a JEM-1200EX transmission electron microscope (TEM) (JEOL, Tokyo, Japan).

\subsection{Estimation of Serum Ferritin, Hepcidin and Iron-Related Protein Expression}

The levels of serum ferritin (SF) and hepcidin (HEPC) were measured by a competitive enzyme immunoassay using ELISA kit, and procedures strictly followed the manufacturer's instructions.

Subsequently, the protein expressions of divalent-metal transporter 1 (DMT1) and ferroportin 1 (FPN1) were performed by western blot analysis. Concisely, the experiment homogenized and lysed the intestine tissues to extract total proteins in radioimmunoprecipitation assay (RIPA) buffer including phenylmethanesulfonyl fluoride (PMSF). A BCA Protein Assay Kit was used to determine protein concentrations. An Enhanced chemiluminescence (ECL) plus system and a Molecular Imager ChemiDoc XRS System (Bio-Rad Laboratories, Hercules, CA, USA) were used to detect bound antibodies. Image Lab 2.0 Software (Bio-Rad Laboratories, Berkeley, CA, USA) identified and quantified optical densities of bands.

\subsection{Measurement of Endotoxin Assay and Intestinal Mucosal Barrier Assay}

CE TAL assay kit was obtained from Limulus Reagent Rlant Corp (Xiamen, China) and used to detect the plasma endotoxin levels. The experimental procedures were conducted following the manufacturer's instructions. ELISA assay kits (Cloud-Clone Corp, Katy, USA) were used to determine D-lactic acid (D-LA), fatty acid-binding protein 2 (FABP2) levels and diamine oxidase (DAO) activity in plasma, in accordance with the instructions of manufacturer. The levels of DAO, D-LA, and FABP2 were identified from a standard curve. The data were reported as $\mathrm{U} \mathrm{L}^{-1}, \mu \mathrm{mol} \mathrm{L}-1$ and $\mathrm{ng} \mathrm{mL}^{-1}$, respectively.

\subsection{Gut Microbiota Analysis}

Genome DNA was extracted from $200 \mathrm{mg}$ feces samples and stored at $-20{ }^{\circ} \mathrm{C}$. The contents of bacterial genome DNA were quantified by using real-time fluorescence quantification polymerase chain reaction (PCR) method, involving Escherichia coil, Enterococcus, Bifidobacterium, Lactobacillus, Bacteroides fragilis and Clostridium tender. The reaction system and reaction condition are similar to 
standard curve. According to the manufacturers' instructions, the whole sequence was amplified for fluorescent real-time PCR with the kit (QIAgen, Shanghai, China). Primer sequences and annealing temperature of PCR amplification of specific bacterial are shown in Table 2.

Table 2. Primer sequences and annealing temperature of PCR amplification of specific bacterial.

\begin{tabular}{|c|c|c|c|}
\hline Bacterial Species & Primer Sequences $\left(5^{\prime} 3^{\prime}\right)$ & Lengths (bp) & Annealing Temperature $\left({ }^{\circ} \mathrm{C}\right)$ \\
\hline Escherichia coil & $\begin{array}{l}\text { F: 5'-GTTAATACCTTTGCTCATTGA-3' } \\
\text { R: 5'-ACCAGGGTATCTTAATCCTGTT-3' }\end{array}$ & 340 & 51 \\
\hline Enterococcus & $\begin{array}{l}\text { F: } 5^{\prime} \text {-ACTCGTTGTACTTCCCATTGT-3' } \\
\text { R:5'-CCCTTATTGTTAGTTGCCATCATT-3' }\end{array}$ & 144 & 52 \\
\hline Bifidobacterium & $\begin{array}{c}\text { F: 5'-GGGTGGTAATGCCGGATG-3' } \\
\text { R: } \text { 5'-TAAGCGATGGACTTTCACACC-3' }^{\prime}\end{array}$ & 442 & 61 \\
\hline Lactobacillus & $\begin{array}{c}\text { F: 5'- AGCAGTAGGGAATCTTCCA-3' } \\
\text { R: } 5^{\prime} \text {-CACCGCTACACATGGAG-3' }\end{array}$ & 341 & 55 \\
\hline Bacteroides fragilis & 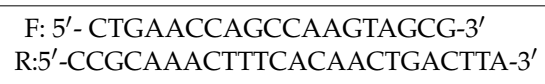 & 230 & 62 \\
\hline Clostridium tender & $\begin{array}{l}\text { F: 5'-GCACAAGCAGTGGAGT-3' } \\
\text { R: 5'-CTTCCTCCGTTTTGTCAA-3' }\end{array}$ & 246 & 58 \\
\hline
\end{tabular}

\subsection{Statistical Analysis}

SPSS 18 (SPSS, Chicago, IL, USA) were used to analyze all data which were expressed as mean \pm standard deviation (SD). One-way analysis of variance (ANOVA) was used to compare multiple groups with a Duncan's multiple range test. It was considered to be statistically significant when results were at $p<0.05$.

\section{Results}

\subsection{Effects of Aplysin on Food Intake and Growth Performance}

The information of the initial and final body weight (BW) was shown in Table 3. The initial body weight and diet intake among groups were no different at the end of 12 weeks $(p>0.05)$. However, the final body weight was decreased in rats treated with ethanol and iron compared with the control group, which was also attenuated by aplysin significantly $(p<0.05)$.

Table 3. Effects of aplysin on food intake and body weight.

\begin{tabular}{cccc}
\hline Group $(\boldsymbol{n}=\mathbf{1 0})$ & Food Intake $(\mathbf{g})$ & Initial Body Weight $(\mathbf{g})$ & Final Body Weight $(\mathbf{g})$ \\
\hline C & $35.93+1.76$ & $233.40+12.35$ & $398.00+12.75$ \\
EI & $34.72+1.36$ & $236.55+8.23$ & $350.30+13.76^{\#}$ \\
AEI & $35.50+1.80$ & $238.00+12.14$ & $379.10+14.91^{\#, *}$ \\
\hline
\end{tabular}

${ }^{\#} p<0.05$ versus $C$ group, ${ }^{*} p<0.05$ versus EI group. C: Control Group; EI: Ethanol and Iron Group; AEI: Aplysin $\left(150 \mathrm{mg} \mathrm{kg}^{-1}\right)$ with Ethanol and Iron Group.

\subsection{Effects of Aplysin on Serum Ferritin and Hepcidin}

Serum ferritin and hepcidin of each group were shown in Figure 2. Chronic consumption of ethanol and iron (EI group) resulted in high levels of ferritin and hepcidin in serum $(p<0.05)$. Conversely, compared with the EI model group, the serum ferritin and hepcidin in the co-treatment of ethanol and iron rats with aplysin treatment were significantly lower $(p<0.05)$. 


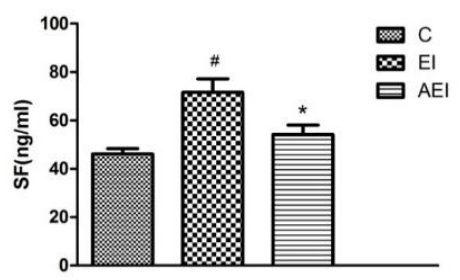

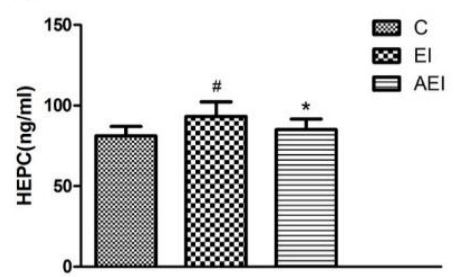

Figure 2. Effects of aplysin on serum ferritin (SF) and hepcidin (HEPC). (A) The serum ferritin in rats. (B) The serum hepcidin in rats. C: Control Group; EI: Ethanol and Iron Group; AEI: Aplysin $\left(150 \mathrm{mg} \mathrm{kg}^{-1}\right)$ with Ethanol and Iron Group. ${ }^{*} p<0.05$ versus $C$ group, ${ }^{*} p<0.05$ versus EI group.

\subsection{Effects of Aplysin on Intestinal Permeability}

As shown in Figure 3, compared with the control group, the level of endotoxin in plasma was significantly increased in the ethanol-iron-challenged rats $(p<0.05)$. Aplysin treatment daily to ethanol and iron-fed rats evidently decreased the endotoxin level $(p<0.05)$ (Figure 3A). Meanwhile, significantly higher DAO activity, and plasma levels of D-LA and FABP2 were observed in the ethanol and iron combined group in contrast with the control group $(p<0.05)$. In the aplysin treatment group, the plasma levels of DAO, D-LA, and FABP2 were reduced by $16 \%, 26 \%, 17 \%$, respectively $(p<0.05)$, compared to those in co-dosed rats.

A

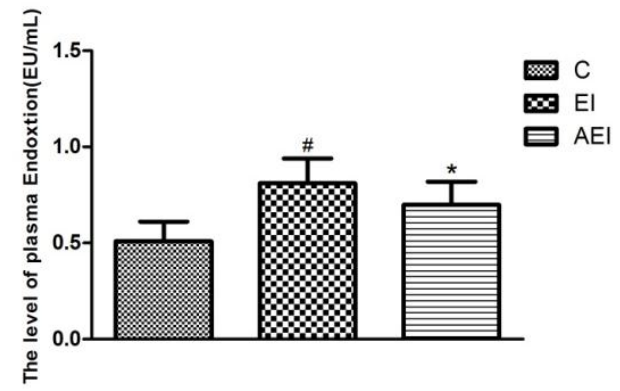

C

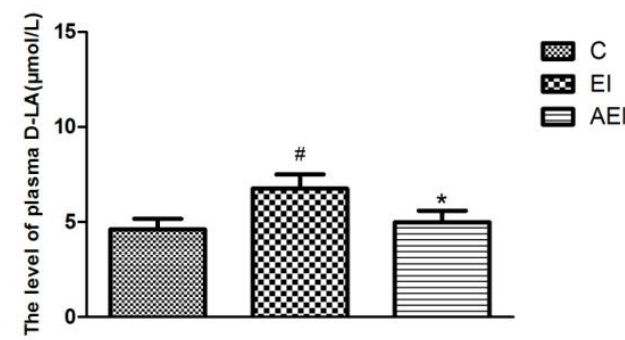

B

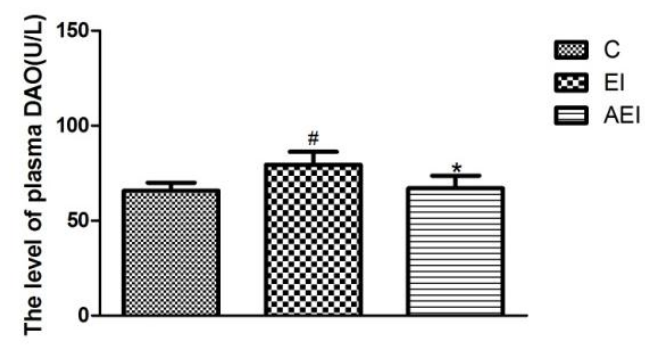

D

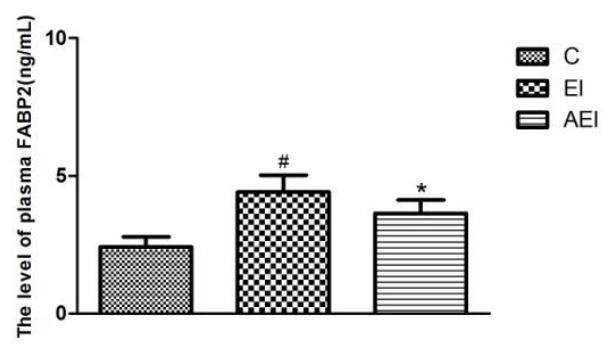

Figure 3. Effects of aplysin on intestinal permeability. (A) The plasma endotoxin level in rats. (B) Plasma diamine oxidase (DAO) level in rats. (C) Plasma D-lactic acid (D-LA ) level in rats. (D) Plasma fatty acid-binding protein 2 (FABP2) level in rats. C: Control Group; EI: Ethanol and Iron Group; AEI: Aplysin $\left(150 \mathrm{mg} \mathrm{kg}^{-1}\right)$ with Ethanol and Iron Group. ${ }^{\#} p<0.05$ versus $\mathrm{C}$ group, ${ }^{*} p<0.05$ versus EI group.

\subsection{Effects of Aplysin on Intestinal Microbiota}

As shown in Figure 4, the results indicated that the amplification products were single after each quantitative PCR reaction. With the increase of cycle number, the fluorescence intensity was also enhanced. The curve tended to be parallel after a period of index amplification, namely a "platform effect" was observed, indicating that the corresponding relation between template copies at the index expansion period and the fluorescence cumulative value was the quantitative basis (Figure 5). 
The status of the intestinal integrity may also be reflected through fecal microbial culture. The amount of Escherichia coil, Enterococcus, Bifidobacterium, Lactobacillus, Bacteroides fragilis and Clostridium tender of different groups were given in Figure 6. In contrast with the control group, the amount of Escherichia coil and Bacteroides fragilis were elevated significantly $(p<0.05)$, but the amount of Bifidobacterium, Lactobacillus and Clostridium tender were all decreased significantly $(p<0.05)$ when co-treated by ethanol and iron. Aplysin effectively decreased the amount of Escherichia coil and Bacteroides fragilis in comparison with co-treatment of ethanol and iron $(p<0.05)$. Conversely, the amount of Bifidobacterium, Lactobacillus and Clostridium tender were obviously reversed by aplysin to ethanol and iron dosed rats. However, no statistical difference in the amount of Enterococcus among various groups was obtained $(p>0.05)$.

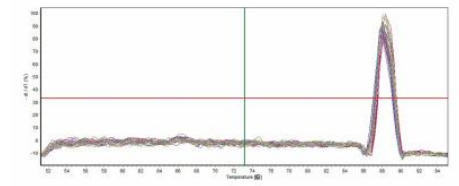

a

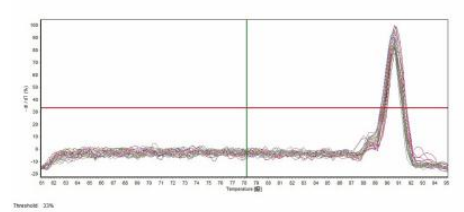

d

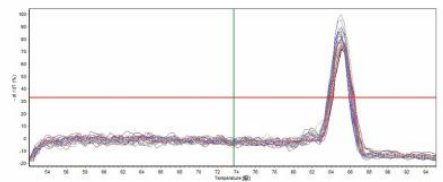

b

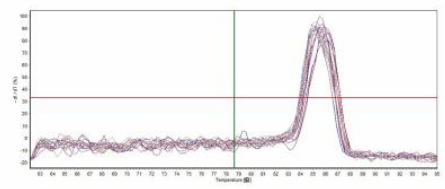

e

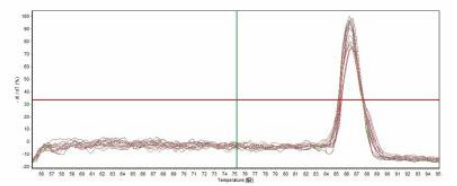

C

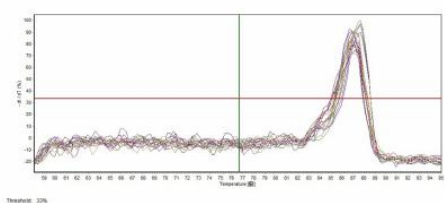

f

Figure 4. Melting curve of intestinal microbiota in different groups of rats by real-time q-PCR. (a) Escherichia coil, (b) Enterococcus, (c) Lactobacillus, (d) Bifidobacterium, (e) Bacteroides fragilis, (f) Clostridium tender.

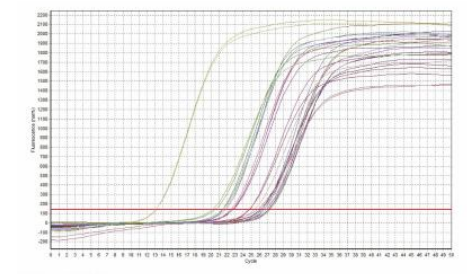

a

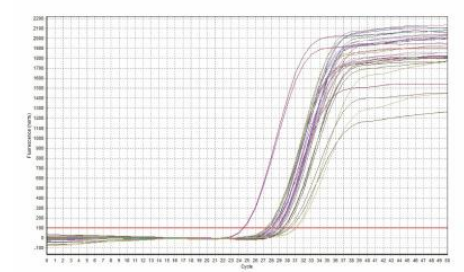

d

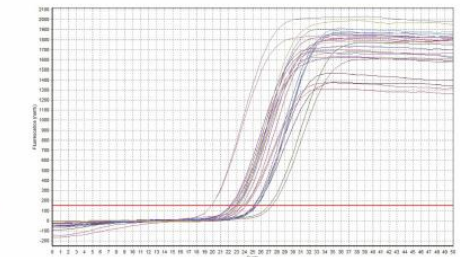
$=-\infty$

b

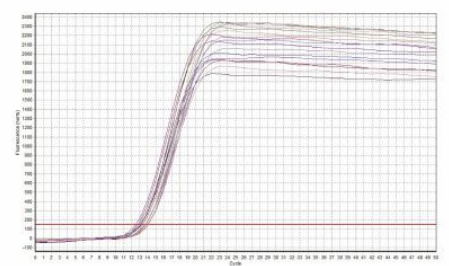

$=-10$

e

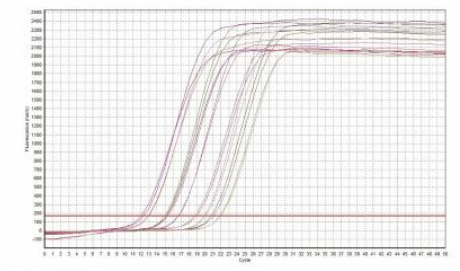

$=-10$

C

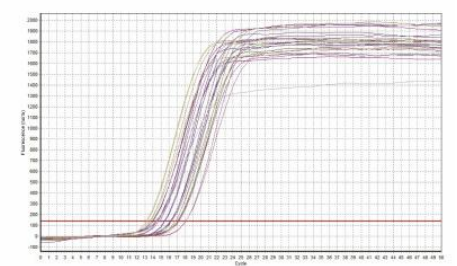

$=-m$

f

Figure 5. Amplification curve of intestinal microbiota in different groups of rats by real-time q-PCR. (a) Escherichia coil, (b) Enterococcus, (c) Lactobacillus, (d) Bifidobacterium, (e) Bacteroides fragilis, (f) Clostridium tender. 


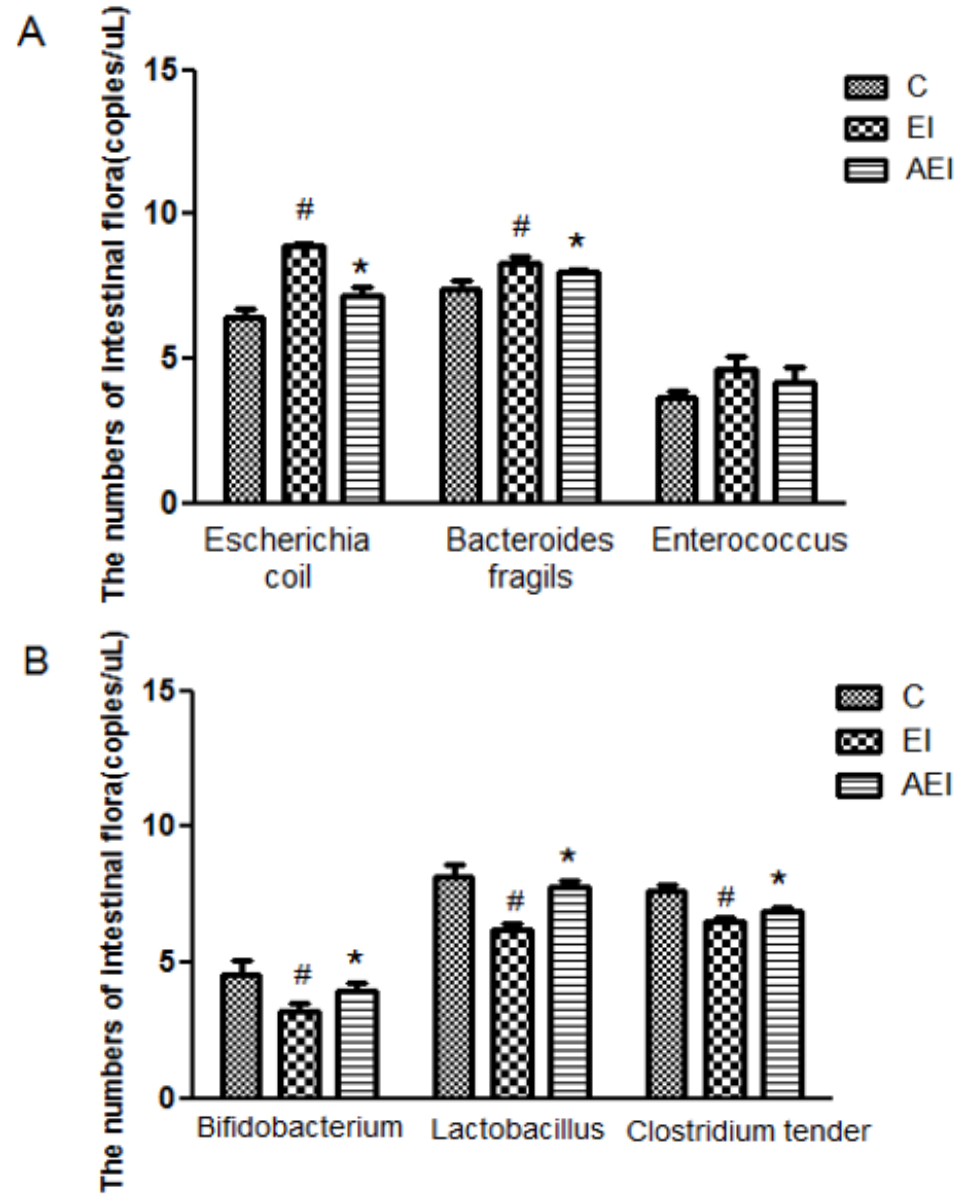

Figure 6. Effects of aplysin on intestinal microbiota. (A) The amount of Escherichia coil, Bacteroides fragilis and Enterococcus in feces of rats. (B) The amount of Bifidobacterium, Lactobacillus and Clostridium tender in feces of rats. C: Control Group; EI: Ethanol and Iron Group; AEI: Aplysin $\left(150 \mathrm{mg} \mathrm{kg}^{-1}\right)$ with Ethanol and Iron Group. ${ }^{\#} p<0.05$ versus $C$ group, ${ }^{*} p<0.05$ versus EI group.

\subsection{Effects of Aplysin on Pathological Changes of Liver and Intestine}

According to HE staining for light microscopy of liver sections in different groups, the control groups showed normal lobular architecture. The hepatic cord was orderly. In addition, there was no steatosis in the liver (Figure 7A). Some extent of liver damage in the ethanol-iron treated group was indicated, such as the liver cells swelling, hepatic cord derangement, inflammatory infiltration, microvesicular steatosis and fat vacuoles (Figure 7B). However, in comparison with the co-treatment of ethanol and iron group, aplysin significantly improved these histopathological changes, and alleviated steatosis in the liver (Figure 7C).

According to HE staining for light microscopy of the intestine section in diverse groups, there was the intact and normal mucosal tissue in the small intestine, and intestinal villi arranged neatly in rats of the control group (Figure 8A). After ethanol-iron exposure, intestinal villus and glands were damaged significantly, mucosal epithelium and lamina propria separation, many mucosal epithelial cells fallen off, and intestinal villus hemorrhage and edema (Figure 8B). However, the intervention of aplysin improved the pathological injuries (Figure 8C).

Further assessment of morphology with transmission electron microscopy (TEM) revealed that the small intestinal epithelial cells were arranged in neat rows. Rich, small intestinal villi and complete tight junctions between cells in the control group were clearly shown (Figure 9A). However, sparse small intestine microvilli and enlarged tight junctions were apparent by co-treatment of ethanol plus iron (Figure 9B). To varying degrees, the arrangement of small intestinal columnar epithelium and 
microvilli in the aplysin treatment group was improved and the swelling of the tight junctions was reduced (Figure 9C).
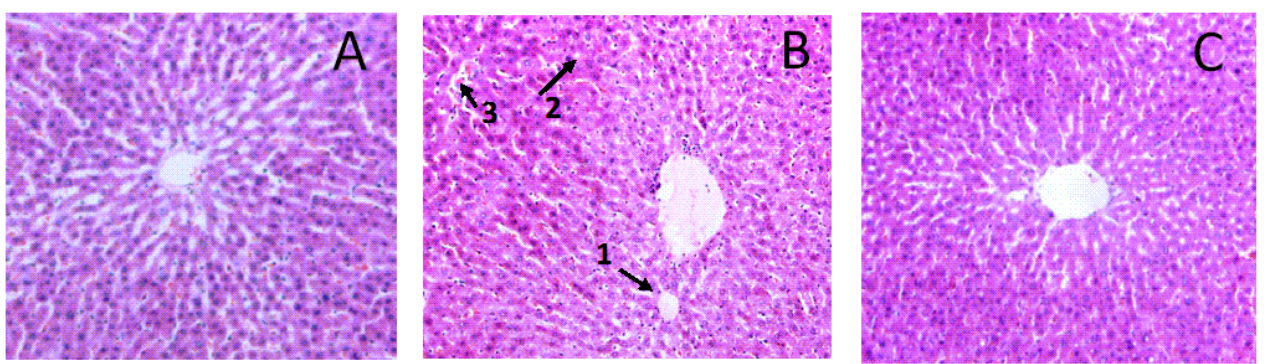

Figure 7. Ethanol-iron-induced liver histopathological changes in rats (Hematoxylino-eosin (HE) Staining, $\times 200$ ). Normal architecture and structural intactness were shown in the control group. Hepatic cord derangement, inflammatory infiltration, microvesicular steatosis were shown in the EI model group. Aplysin $\left(150 \mathrm{mg} \mathrm{kg}^{-1}\right)$ treatment showed the architecture is almost normal. 1: Fat vacuoles; 2: Hepatocyte microvesicular steatosis; 3: Inflammatory infiltration. (A) Control Group; (B) Ethanol and Iron Group; (C) Aplysin (150 mg kg-1) with Ethanol and Iron Group.
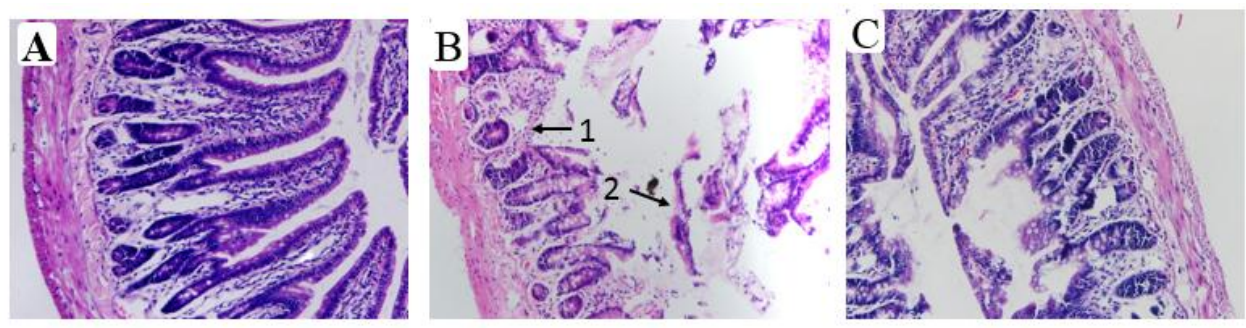

Figure 8. The pathological changes in the small intestine in rats (Hematoxylino-eosin (HE) Staining, $\times 200$ ). It is indicated from HE staining that the mucosal tissue in the small intestine were normal and intact, and all intestinal villi were arranged neatly in rats of the control group. After ethanol-iron exposure, intestinal villus and glands were damaged significantly and many epithelial cells of small intestinal mucosa had fallen off. The pathological damages were improved after treated with aplysin. 1: Intestinal villus and glands were damaged significantly; 2 : Large number of mucosal epithelial cell falls off. (A) Control Group; (B) Ethanol and Iron Group; (C) Aplysin $\left(150 \mathrm{mg} \mathrm{kg}^{-1}\right)$ with Ethanol and Iron Group.

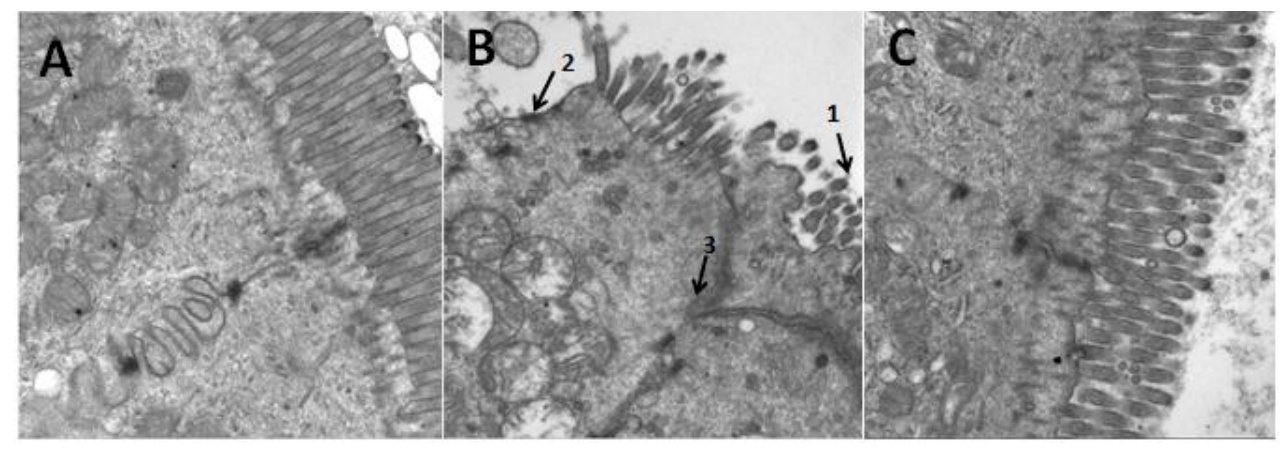

Figure 9. The ultrastructure of small intestine of rats (Magnification, $\times 20,000$ ). In TEM, the tight junctions of the epithelial cells of intestine in the EI group were destroyed while the alterations of tight junctions in aplysin administrated rats were apparent. 1: Small intestine microvillis were sparse; 2: Small intestine microvillis fell away; 3 : The cell connection structures were incomplete even disappear. (A) Control Group; (B) Ethanol and Iron Group; (C) Aplysin (150 mg kg-1) with Ethanol and Iron Group. 


\subsection{Effects of Aplysin on Expressions of Iron-Related Proteins in the Intestine}

The expressions of FPN1 and DMT1 proteins were up-regulated after co-treatment of ethanol plus iron compared to the control group ( $p<0.05$, Figure 10). Importantly, the expressions of FPN1 and DMT1 were clearly down regulated after aplysin treatment by $29 \%$ and $26 \%(p<0.05)$.
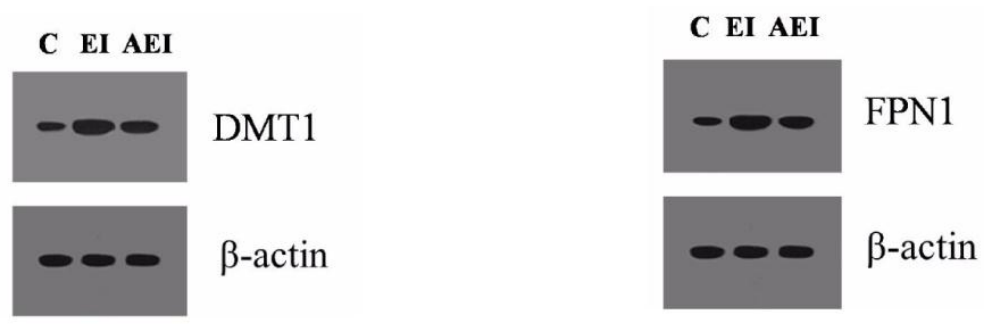

A

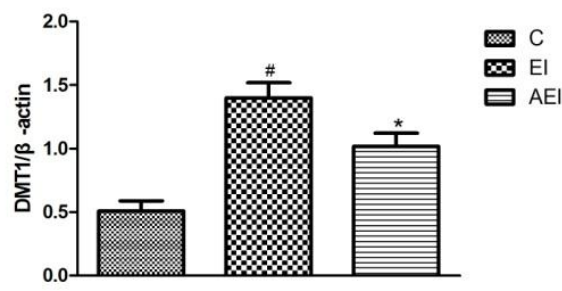

B

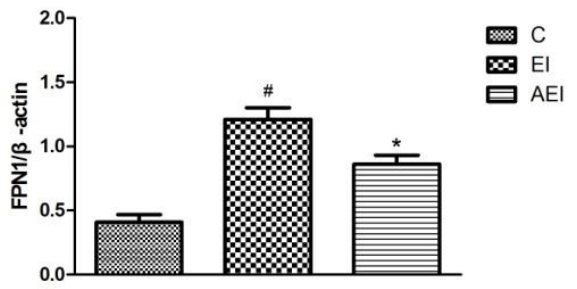

Figure 10. The protein expressions of divalent-metal transporter 1 (DMT1) and ferroportin 1 (FPN1). (A) The expression of divalent-metal transporter 1(DMT1); (B) The expression of divalent-metal transporter 1 (FPN1). C: Control Group; EI: Ethanol and Iron Group; AEI: Aplysin $\left(150 \mathrm{mg} \mathrm{kg}^{-1}\right)$ with Ethanol and Iron Group. ${ }^{\#} p<0.05$ versus $C$ group, ${ }^{*} p<0.05$ versus EI group.

\section{Discussion}

By a 12-week treatment with ethanol and iron in rats, our study demonstrated that supplementary aplysin significantly improved the histopathological damages and enhanced the intestinal mucosal barrier and maintained intestinal permeability. Our study found that an effective treatment may be associated with altered intestinal microflora. These results suggested that the development of intestinal damage was significantly prevented and the intestinal integrity was protected by aplysin.

We previously found that a continuous increase in the dose of alcohol $\left(8-12 \mathrm{~mL} \mathrm{~kg}^{-1}\right.$ day $\left.^{-1}\right)$ caused damage in hepatic cells and it could overcome the tolerance induced by the same dose consumption of ethanol [13]. Ge et al. also reported that aplysin had the hepatoprotective effect on oxidative damage caused by ethanol, which could prevent alcoholic liver injury [13]. Xue et al. showed that aplysin could protect ethanol-induced hepatic injury, repair barrier function of the intestine and provide a low inflammatory response and a low level of plasma endotoxin [15]. Another study from a Chinese journal showed that the intestinal mucosal damage and lipid metabolism disorder caused by excessive iron exposure in rats were protected by aplysin [16]. Therefore, the current study further investigates the protective effects of aplysin on gut microbiota and intestinal permeability in rats co-treated with ethanol and iron.

The present study found that the final body weight was significantly lower in co-treatment of ethanol and iron in contrast with the control group, although each group had the same diet intake. It indicated that nutrition intake and absorption were affected, and the efficiency of calorie utilization was reduced in rats treated with ethanol and iron in a long-term period [17].

Intestinal health was investigated and discussed by measuring the barrier function of intestinal mucosa and the level of plasma endotoxin. Alcohol induced the damage to the barrier function of intestinal mucosa, increased intestinal permeability resulted from endotoxin and other adverse factors, and contributed to the endotoxemia in alcoholic liver damage $[15,18]$. In this study, the plasma endotoxin level was significantly higher in the co-treatment of ethanol plus iron group. However, 
ethanol and iron exposure with aplysin intervention showed that the plasma endotoxin level was lower than that in the model group (EI group). It suggested that aplysin may inhibit the high intestinal permeability induced by combined treatment of ethanol and iron.

The data in this study demonstrated that the D-LA, DAO and FABP2 levels in plasma were increased by long-term intake of ethanol and iron. DAO and D-LA are prevented from infiltratrating the portal blood, providing a barrier function by intact intestinal mucosa, so they are indices of high intestinal permeability [19]. Some studies documented that DAO activity and the D-LA level in the chronic ethanol intake were significantly increased $[15,20]$. Furthermore, decreasing the DAO activities and D-LA and FABP2 levels in plasma were observed after aplysin administration in the study. It showed that aplysin treatment could protect intestinal permeability and barrier function of intestinal mucosa in rats with ethanol and iron exposure.

Normal intestine permeability and the gut microbes are important factors for intestinal health. Fecal microbes are usually used to reflect gut microbes. The changes of intestinal microbiota and the proportion and number of bacteria in the small intestine were caused by alcohol intake, which was relative to ecological imbalance [5]. Moreover, intestinal dysbacteriosis resulted in deficiency of necessary micronutrients such as short chain fatty acids and others in the mucosa. It also led to the intestinal mucosal barrier being damaged and the intestinal permeability being increased [21]. It has been documented that the most frequent gut microbes in the intestinal tract are species of Bacteroides fragilis, Porphyromonas, Bifidobacterium, Lactobacillus, Clostridium tender, and Escherichia coil [22]. The results in this study showed a significantly higher amount of Escherichia coil and Bacteroides fragilis and a lower amount of Bifidobacterium, Lactobacillus and Clostridium tender when co-treated by ethanol and iron.

It has been suggested that Bacteroides can damage the intestinal health and may be carcinogenic [23]; it could use intestinal macrophages to stimulate the anti-inflammatory cytokine interleukin (IL)-10 production, as the most powerful inducer of regulatory $\mathrm{T}$ cells [24]. It has been reported that systemic and mucosal immune responses were modulated by certain Bifidobacterium and Lactobacillus species [25]. Lactobacillus has some positive physiological effects, which ferments carbohydrates to produce lactic acid. It can stop some harmful bacteria from the epithelial cells of the intestine and improve intestinal epithelial cells barrier function. It also can stimulate immunoglobulin production and strengthen the immune system of the host [26]. Our previous study showed that alcohol intake decreased the abundances of Lactobacillus [15]. Another study also suggested that the numbers of fecal Bifidobacterium were reduced, but Escherichia coil were increased in the ethanol group [27]. In a previous study, in rats of alcoholic steatohepatitis, it indicated that Lactobacillus GG treatment improved liver injury, gut leakiness and intestinal oxidative stress induced by alcohol [28]. Moreover, a study revealed that Fe deficiency increased the numbers of Lactobacillus with a trend towards decreased Bacteroides during in vitro colonic fermentation [29]. Zimmermann et al. investigated gut microbiota of African school children Fe supplemented for six months. It concluded that there were higher concentrations of Enterobacteriaceae and a lower amount of Lactobacilli in fecal samples of children with Fe supplemented biscuits which contained $20 \mathrm{mg}$ Fe/d, compared to a control group with normal biscuits. A potentially more pathogenic gut microbiota profile which was produced by iron fortification is more related to increased gut inflammation [30]. In the present study, we also found that aplysin treatment produced a lower amount of Escherichia coil and Bacteroides fragilis and a higher amount of Bifidobacterium, Lactobacillus and Clostridium tender in feces and may have a tendency to restore the structure of intestinal flora in rats with combined treatment of ethanol and iron.

Serum ferritin and hepcidin, key regulators of iron metabolism, were detected in our study. The levels of serum hepcidin and ferritin significantly increased in co-treatment with ethanol and iron group compared with control. There was a similar result in which hepcidin protein and mRNA expression were higher in rats combined with ethanol and iron compared with the control group [31]. It suggested that excessive carbonyl iron caused primary iron overload, resulting in the high level of hepcidin expression. In contrast, chronic ethanol consumption decreased hepcidin expression, 
demonstrating that ethanol did not completely block the hepcidin expression induced by carbonyl iron [31]. Ioannou et al. suggested that chronic consumption of alcohol causes increased ferritin concentration and serum transferrin saturation, and even elevated iron stores in the liver [32]. Moreover, patients with chronic alcoholic disease exhibited increased intestinal iron absorption [33]. It was documented that the levels of serum iron, serum ferritin and transferrin saturation were increased gradually with the increases of iron levels in the diet [34]. Both ethanol and iron separately resulted in lipid peroxidation and oxidative stress, and damaged liver cells by the cumulative effects of iron and ethanol, even exacerbating liver injury in patients with alcoholic liver disease [35]. The results from the present study showed that aplysin significantly decreased serum ferritin and hepcidin induced by combined treatment of ethanol and iron.

Under HE and TEM observation, aplysin intervention alleviated fat accumulation and inflammation in the liver and the intestinal epithelial cells destroyed, and improved the pathological injuries, as previously suggested [15]. In recent years our understanding about intestinal iron absorption has improved by the identification of DMT1 and FPN1 [36]. We, therefore, found that DMT1 and FPN1 in the intestine were significantly upregulated after 12 weeks feeding with ethanol and iron. Harrison-Findik has hypothesized that chronic alcohol metabolism can result in down-regulating hepcidin expression, increasing intestinal absorption, and iron overload [37]. It has been documented that alcohol-mediated hepcidin suppression in the liver leads to high expression of ferroportin 1 and DMT1 in the duodenum [38,39]. It is possible that elevated ferroportin 1 expression in enterocytes may induce iron depletion, leading to the up-regulation of DMT1 [38]. Another study found similar results in that alcohol consumption decreased the serum hepcidin levels and led to elevation of DMT1 at the mRNA level and iron transporter ferroportin at the levels of mRNA and protein in the duodenum of patients with alcoholic liver disease [40]. There is a study showing that duodenal DMT1 levels in iron overloaded group were lower compared with the iron adequate group [41]. Harrison-Findik investigated the influence of iron together with alcohol in the intestine [39]. They demonstrated that the ferroportin expression in duodenum in the co-treated with ethanol and iron group elevated and reached the levels between iron-exposed and ethanol-exposed rats. It also observed that the down-regulation of hepcidin mRNA expression mediated by alcohol resulted in elevated expression of duodenal ferroportin in the iron-overloaded group. It has been shown that hepcidin binds to and degrades ferroportin protein, inhibiting iron uptake and release [42]. Except for in the iron-overloaded group, alcohol was shown to suppress iron-induced hepcidin expression, leading to increasing iron transport, as concluded by Harrison-Findik [39]. To our knowledge, it is the first time that aplysin treatment has been reported to evidently suppress the up-regulation of intestinal DMT1 and FPN1 expressions induced by co-treatment of ethanol plus iron.

However, the mechanisms between maintenance of microbiota composition and the barrier of intestinal mucosa are complicated and diverse. The proportion of different bacterial species or the intestinal flora composition plays a critical role in many cases. We should accurately analyze the fecal microflora both in quantitative techniques and in flora species. Therefore, analysis of the bacterial composition through $16 \mathrm{~S}$ ribosomal DNA high throughput sequencing will be needed in our future studies.

\section{Conclusions}

The present study suggested that chronic co-treatment of ethanol plus iron could cause a variety of changes in the intestinal flora. The intestinal permeability was increased, the intestinal mucosal barrier was damaged and fecal microbial composition was imbalanced, which then contributed to a high plasma endotoxin level in rats as well as intestinal damage. However, aplysin intervention under ethanol and iron exposure could normalize fecal microflora composition, intestinal permeability and intestinal barrier function, and alleviate the high plasma levels of endotoxin, which showed a protective effect on ethanol-iron induced damage. These new findings suggest the rational use of marine food resources and provide a scientific basis for improving human health. 
Author Contributions: H.L., Y.L. and Y.M. conceived and designed the experiments; Y.M., M.L. and R.L. performed experiments and analyzed the data. Hui Liang and Y.L. were responsible for the critical review of the study design, protocol, and methodology, providing scientific advice to this study. Y.M. and R.L. contributed to interpreting the data as well as writing and editing the manuscript. All authors approved the final version of the manuscript.

Acknowledgments: This work was funded and supported by National Natural Science Foundation of China (Grant No. 81573137, Grant No. 31171671), Key Research and Development Program in Shandong Province of China (Grant No. 2017GSF18167) and Medical Science and Technology Development Program in Shandong Province of China (Grant No. 2016WS0254).

Conflicts of Interest: The authors declare no conflict of interest.

\section{References}

1. Galaris, D.; Pantopoulos, K. Oxidative stress and iron homeostasis: Mechanistic and health aspects. Crit. Rev. Clin. Lab. Sci. 2008, 45, 1-23. [CrossRef] [PubMed]

2. Tsuchiya, H.; Ebata, Y.; Sakabe, T.; Hama, S.; Kogure, K.; Shiota, G. High-fat, high-fructose diet induces hepatic iron overload via a hepcidin-independent mechanism prior to the onset of liver steatosis and insulin resistance in mice. Metabolism 2013, 62, 62-69. [CrossRef] [PubMed]

3. Pascoe, A.; Kerlin, P.; Steadman, C.; Clouston, A.; Jones, D.; Powell, L.; Jazwinska, E.; Lynch, S.; Strong, R. Spur cell anaemia and hepatic iron stores in patients with alcoholic liver disease undergoing orthotopic liver transplantation. Gut 1999, 45, 301-305. [CrossRef] [PubMed]

4. Fletcher, L.M.; Halliday, J.W.; Powell, L.W. Interrelationships of alcohol and iron in liver disease with particular reference to the iron-binding proteins, ferritin and transferrin. J. Gastroenterol. Hepatol. 1999, 14, 202-214. [CrossRef] [PubMed]

5. Yan, A.W.; Fouts, D.E.; Brandl, J.; Starkel, P.; Torralba, M.; Schott, E.; Tsukamoto, H.; Nelson, K.E.; Brenner, D.A.; Schnabl, B. Enteric dysbiosis associated with a mouse model of alcoholic liver disease. Hepatology 2011, 53, 96-105. [CrossRef] [PubMed]

6. Chen, Y.; Yang, F.; Lu, H.; Wang, B.; Chen, Y.; Lei, D.; Wang, Y.; Zhu, B.; Li, L. Characterization of fecal microbial communities in patients with liver cirrhosis. Hepatology 2011, 54, 562-572. [CrossRef] [PubMed]

7. Qin, X.; Deitch, E.A. Dissolution of lipids from mucus: A possible mechanism for prompt disruption of gut barrier function by alcohol. Toxicol. Lett. 2015, 232, 356-362. [CrossRef] [PubMed]

8. Kirpich, I.A.; Feng, W.; Wang, Y.; Liu, Y.; Beier, J.I.; Arteel, G.E.; Falkner, K.C.; Barve, S.S.; McClain, C.J. Ethanol and dietary unsaturated fat (corn oil/linoleic acid enriched) cause intestinal inflammation and impaired intestinal barrier defense in mice chronically fed alcohol. Alcohol 2013, 47, 257-264. [CrossRef] [PubMed]

9. Kishikawa, H.; Miura, S.; Nishida, J.; Nakano, M.; Hirano, E.; Sudo, N.; Morishita, T.; Ishii, H. Ethanol-induced CXC-chemokine synthesis and barrier dysfunction in intestinal epithelial cells. Alcohol. Clin. Exp. Res. 2005, 29, 2116-2122. [CrossRef] [PubMed]

10. Elamin, E.; Masclee, A.; Dekker, J.; Jonkers, D. Ethanol disrupts intestinal epithelial tight junction integrity through intracellular calcium-mediated Rho/ROCK activation. Am. J. Physiol. Gastrointest. Liver Physiol. 2014, 306, G677-G685. [CrossRef] [PubMed]

11. Summa, K.C.; Voigt, R.M.; Forsyth, C.B.; Shaikh, M.; Cavanaugh, K.; Tang, Y.; Vitaterna, M.H.; Song, S.; Turek, F.W.; Keshavarzian, A. Disruption of the Circadian Clock in Mice Increases Intestinal Permeability and Promotes Alcohol-Induced Hepatic Pathology and Inflammation. PLoS ONE 2013, 8, e67102. [CrossRef] [PubMed]

12. Fletcher, L.M.; Powell, L.W. Hemochromatosis and alcoholic liver disease. Alcohol 2003, 30, 131-136. [CrossRef]

13. Ge, N.; Liang, H.; Liu, Y.; Ma, A.G.; Han, L. Protective effect of Aplysin on hepatic injury in ethanol-treated rats. Food Chem. Toxicol. 2013, 62, 361-372. [CrossRef] [PubMed]

14. He, J.; Liang, H.; Li, Y.; Shi, D.Y.; Ma, A.G. Antioxidant effect of Aplysin on aged mice exposed to D-galatose. Chin. J. Public Health 2009, 25, 1122-1123.

15. Xue, M.; Liu, Y.; Lyu, R.; Ge, N.; Liu, M.; Ma, Y.; Liang, H. Protective effect of aplysin on liver tissue and the gut microbiota in alcohol-fed rats. PLoS ONE 2017, 12, e0178684. [CrossRef] [PubMed] 
16. Li, R.Y.; Ma, Y.; Liu, Y.; Liu, M.; Liang, H. Protective effects of Aplysin on lipid metabolism and intestinal mucosal damage of rats exposed by excessive iron. Chin. J. Mar. Drugs 2017, 36, 54-60.

17. Lieber, C.S. Hepatic, metabolic and toxic effects of ethanol: 1991 update. Alcohol. Clin. Exp. Res. 1991, 15, 573-592. [CrossRef] [PubMed]

18. Rao, R. Endotoxemia and gut barrier dysfunction in alcoholic liver disease. Hepatology 2009, 50, 638-644. [CrossRef] [PubMed]

19. Smith, S.M.; Eng, R.H.; Campos, J.M.; Chmel, H. D-lactic acid measurements in the diagnosis of bacterial infections. J. Clin. Microbiol. 1989, 27, 385-388. [CrossRef] [PubMed]

20. Li, H.; Qiu, P.; Wang, J.; Niu, C.; Pan, S. Effects of compound Ginkgo biloba on intestinal permeability in rats with alcohol-induced liver injury. Food Funct. 2015, 6, 470-478. [CrossRef] [PubMed]

21. Verma, R.; Verma, A.K.; Ahuja, V.; Paul, J. Real-time analysis of mucosal flora in patients with inflammatory bowel disease in India. J. Clin. Microbiol. 2010, 48, 4279-4282. [CrossRef] [PubMed]

22. Jarvelainen, H.A.; Fang, C.; Ingelman-Sundberg, M.; Lindros, K.O. Effect of chronic coadministration of endotoxin and ethanol on rat liver pathology and proinflammatory and anti-inflammatory cytokines. Hepatology 1999, 29, 1503-1510. [CrossRef] [PubMed]

23. Sobhani, I.; Tap, J.; Roudot-Thoraval, F.; Roperch, J.P.; Letulle, S.; Langella, P.; Corthier, G.; Tran Van Nhieu, J.; Furet, J.P. Microbial dysbiosis in colorectal cancer (CRC) patients. PLoS ONE 2011, 6, e16393. [CrossRef] [PubMed]

24. Atarashi, K.; Tanoue, T.; Oshima, K.; Suda, W.; Nagano, Y.; Nishikawa, H.; Fukuda, S.; Saito, T.; Narushima, S.; Hase, K.; et al. Treg induction by a rationally selected mixture of Clostridia strains from the human microbiota. Nature 2013, 500, 232-236. [CrossRef] [PubMed]

25. Blum, S.; Haller, D.; Pfeifer, A.; Schiffrin, E.J. Probiotics and immune response. Clin. Rev. Allergy Immunol. 2002, 22, 287-309. [CrossRef] [PubMed]

26. Kikuchi, Y.; Kunitoh-Asari, A.; Hayakawa, K.; Imai, S.; Kasuya, K.; Abe, K.; Adachi, Y.; Fukudome, S.; Takahashi, Y.; Hachimura, S. Oral administration of Lactobacillus plantarum strain AYA enhances IgA secretion and provides survival protection against influenza virus infection in mice. PLOS ONE 2014, 9, e86416. [CrossRef] [PubMed]

27. Chiu, W.C.; Huang, Y.L.; Chen, Y.L.; Peng, H.C.; Liao, W.H.; Chuang, H.L.; Chen, J.R.; Yang, S.C. Synbiotics reduce ethanol-induced hepatic steatosis and inflammation by improving intestinal permeability and microbiota in rats. Food Funct. 2015, 6, 1692-1700. [CrossRef] [PubMed]

28. Forsyth, C.B.; Farhadi, A.; Jakate, S.M.; Tang, Y.; Shaikh, M.; Keshavarzian, A. Lactobacillus GG treatment ameliorates alcohol-induced intestinal oxidative stress, gut leakiness, and liver injury in a rat model of alcoholic steatohepatitis. Alcohol 2009, 43, 163-172. [CrossRef] [PubMed]

29. Dostal, A.; Fehlbaum, S.; Chassard, C.; Zimmermann, M.B.; Lacroix, C. Low iron availability in continuous in vitro colonic fermentations induces strong dysbiosis of the child gut microbial consortium and a decrease in main metabolites. FEMS Microbiol. Ecol. 2013, 83, 161-175. [CrossRef] [PubMed]

30. Zimmermann, M.B.; Chassard, C.; Rohner, F.; N'Goran, E.K.; Nindjin, C.; Dostal, A.; Utzinger, J.; Ghattas, H.; Lacroix, C.; Hurrell, R.F. The effects of iron fortification on the gut microbiota in African children: A randomized controlled trial in Cote d'Ivoire. Am. J. Clin. Nutr. 2010, 92, 1406-1415. [CrossRef] [PubMed]

31. Tang, Y.; Li, Y.; Yu, H.; Gao, C.; Liu, L.; Chen, S.; Xing, M.; Liu, L.; Yao, P. Quercetin prevents ethanol-induced iron overload by regulating hepcidin through the BMP6/SMAD4 signaling pathway. J. Nutr. Biochem. 2014, 25, 675-682. [CrossRef] [PubMed]

32. Ioannou, G.N.; Dominitz, J.A.; Weiss, N.S.; Heagerty, P.J.; Kowdley, K.V. The effect of alcohol consumption on the prevalence of iron overload, iron deficiency, and iron deficiency anemia. Gastroenterology 2004, 126, 1293-1301. [CrossRef] [PubMed]

33. Duane, P.; Raja, K.B.; Simpson, R.J.; Peters, T.J. Intestinal iron absorption in chronic alcoholics. Alcohol Alcohol. 1992, 27, 539-544. [PubMed]

34. Han, W.; Wang, C.; Su, C.; Xu, X. Effect of higher iron in diet on iron levels and hepcidin mRNA expression levels in rats. Wei Sheng Yan Jiu 2008, 37, 474-476. [PubMed]

35. Sumida, Y.; Nakashima, T.; Yoh, T.; Kakisaka, Y.; Nakajima, Y.; Ishikawa, H.; Mitsuyoshi, H.; Okanoue, T.; Nakamura, H.; Yodoi, J. Serum thioredoxin elucidates the significance of serum ferritin as a marker of oxidative stress in chronic liver diseases. Liver 2001, 21, 295-299. [CrossRef] [PubMed] 
36. Zoller, H.; Koch, R.O.; Theurl, I.; Obrist, P.; Pietrangelo, A.; Montosi, G.; Haile, D.J.; Vogel, W.; Weiss, G. Expression of the duodenal iron transporters divalent-metal transporter 1 and ferroportin 1 in iron deficiency and iron overload. Gastroenterology 2001, 120, 1412-1419. [CrossRef] [PubMed]

37. Harrison-Findik, D.D. Is the iron regulatory hormone hepcidin a risk factor for alcoholic liver disease? World J. Gastroenterol. 2009, 15, 1186-1193. [CrossRef] [PubMed]

38. Harrison-Findik, D.D.; Schafer, D.; Klein, E.; Timchenko, N.A.; Kulaksiz, H.; Clemens, D.; Fein, E.; Andriopoulos, B.; Pantopoulos, K.; Gollan, J. Alcohol metabolism-mediated oxidative stress down-regulates hepcidin transcription and leads to increased duodenal iron transporter expression. J. Biol. Chem. 2006, 281, 22974-22982. [CrossRef] [PubMed]

39. Harrison-Findik, D.D.; Klein, E.; Crist, C.; Evans, J.; Timchenko, N.; Gollan, J. Iron-mediated regulation of liver hepcidin expression in rats and mice is abolished by alcohol. Hepatology 2007, 46, 1979-1985. [CrossRef] [PubMed]

40. Dostalikova-Cimburova, M.; Balusikova, K.; Kratka, K.; Chmelikova, J.; Hejda, V.; Hnanicek, J.; Neubauerova, J.; Vranova, J.; Kovar, J.; Horak, J. Role of duodenal iron transporters and hepcidin in patients with alcoholic liver disease. J. Cell Mol. Med. 2014, 18, 1840-1850. [CrossRef] [PubMed]

41. Giorgi, G.; Roque, M.E. Iron overload induces changes of pancreatic and duodenal divalent metal transporter 1 and prohepcidin expression in mice. Acta Histochem. 2014, 116, 354-362. [CrossRef] [PubMed]

42. Nemeth, E.; Tuttle, M.S.; Powelson, J.; Vaughn, M.B.; Donovan, A.; Ward, D.M.; Ganz, T.; Kaplan, J. Hepcidin regulates cellular iron efflux by binding to ferroportin and inducing its internalization. Science 2004, 306, 2090-2093. [CrossRef] [PubMed]

(C) 2018 by the authors. Licensee MDPI, Basel, Switzerland. This article is an open access article distributed under the terms and conditions of the Creative Commons Attribution (CC BY) license (http://creativecommons.org/licenses/by/4.0/). 